\title{
Advances in Materials Characterization through Digital Microscopy
}

\author{
S. Paciornik, M.H.P. Mauricio, J.R.M. d'Almeida, J.M. Moraes Jr. and C.M. Reis \\ Department of Materials Science and Metallurgy, PUC-Rio, P.O. Box 38008, Rio de Janeiro, RJ, \\ 22453-900, Brazil.
}

Digital Microscopy is the integration between computer-controlled microscopes and image analysis software. Motorized sample holders allow accurate $x-y-z$ specimen positioning with absolute coordinates. This leads to several possibilities such as automatic field scanning, autofocus, mosaic images, extended focus and co-site microscopy. Sophisticated characterization procedures can be fully automated. Some of these possibilities, employing optical microscopy, are reported below.

Figure 1 shows a mosaic image composed of $7 \times 3$ fields of a fiber-reinforced polymer-matrix composite. The larger white rectangle shows the original field size at this magnification. This composite is fabricated by filament winding and one of the primary concerns is the presence of voids. In this view most voids are approximately round, but in other cross-sections voids appear as elongated objects that can exceed the size of a field, even at low mag. Because of the complexity of fiber lay-up, it is very important to get a global view of representative cross-sections of the material to reveal the fraction, shape and spatial distribution of voids. Automatic segmentation followed by shape analysis allowed the measurement of void fraction, size and shape distribution.

Figure 2 shows an image sequence of dentinary surface exposed to chemical etching for different times. In this co-site microscopy experiment, a set of images at specific $\mathrm{x}-\mathrm{y}$ coordinates were captured, the motorized sample holder was lowered to allow in-situ chemical etching, another set of images at the same coordinates were captured and the process was repeated for several etching times. As etching progressed, the dentinary tubules got more and more exposed, and measuring their size and shapes allowed the study of the etching kinetics. As an example, the plot below the images shows the increase in tubule area fraction over etching time, for two different etchants.

When etching in situ is not possible, another approach to co-site microscopy must be used. The sample is glued to a glass slide that fits snugly in the microscope holder. An image acquisition sequence is performed, the sample is removed from the microscope for etching and put back for the next sequence. This is illustrated in Figure 3 for a nodular cast iron sample. Before etching, the graphite nodules stand out against the bright ferrite/perlite matrix and the graphite fraction, size and shape distribution can be automatically obtained. After etching with Nital, ferrite remains bright and can be automatically segmented from the darker graphite and perlite. In traditional metallography, the area fractions before and after etching are never measured in the same fields. With the digital co-site microscopy approach, the exact same fields are evaluated. In this case, mechanical limitations lead to small displacements between images acquired at the same nominal coordinates. An automatic cross-correlation approach allows the registration of images before the final measurements [1].

References

[1] The support of CNPq, Brazilian Research Council is gratefully acknowledged. 


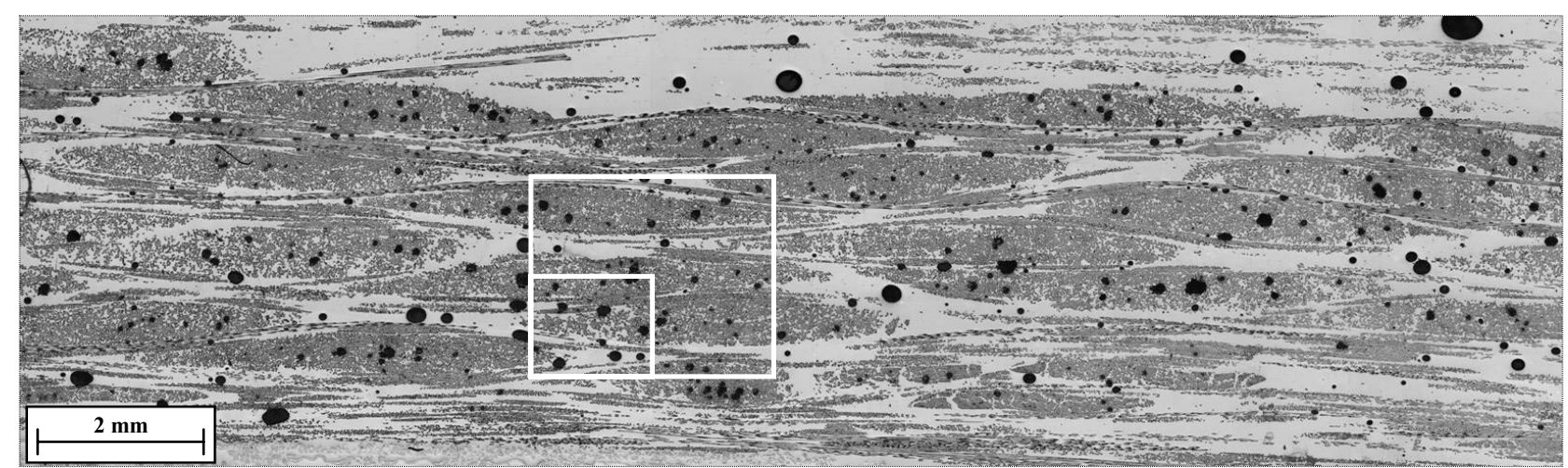

FIG. 1. Mosaic image of a composite material. Large white rectangle shows a single field

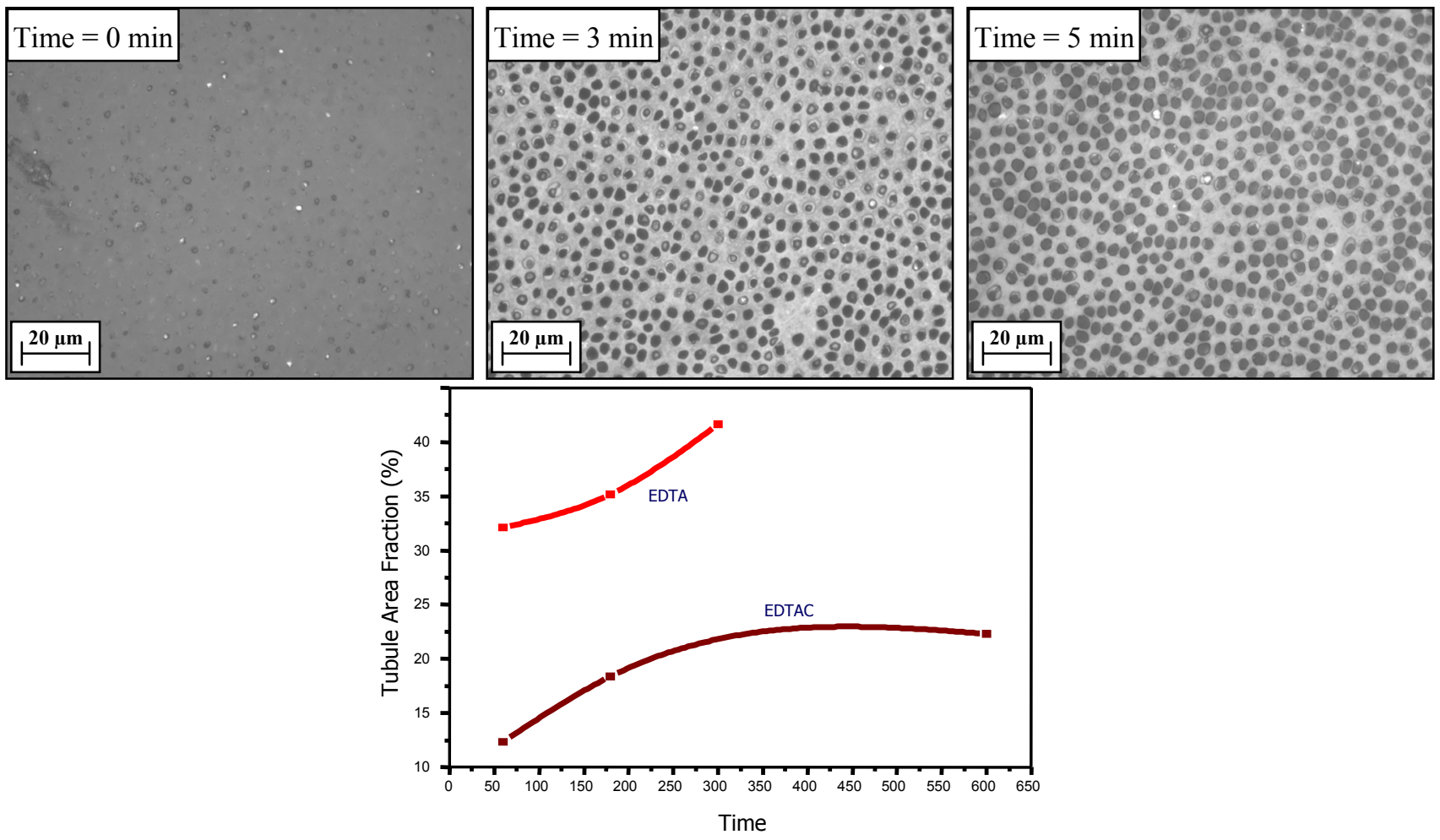

FIG. 2. Chemical etching sequence of dentinary surface, obtained in situ through co-site microscopy. Etching kinetics for two different chemicals.
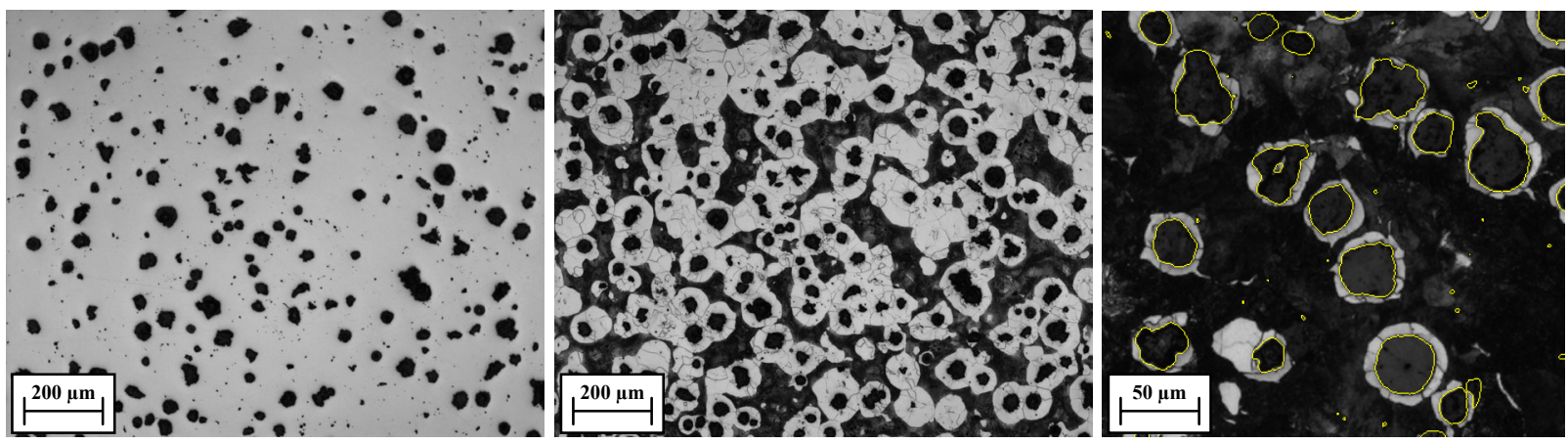

FIG. 3. Co-site characterization of cast iron. (a) Unetched sample. (b) Etched sample. (c) Graphite edges from (a) superimposed on (b). Notice accurate registration. 